\title{
Influences of Silver-Doping on the Crystal Structure, Morphology and Photocatalytic Activity of $\mathrm{TiO}_{2}$ Nanofibers
}

\author{
Nasser A. M. Barakat ${ }^{1,2^{*}}$, Muzafar A. Kanjwal ${ }^{3}$, Salem S. Al-Deyab ${ }^{4}$, Ioannis S. Chronakis ${ }^{3}$, \\ Hak Yong Kim ${ }^{1}$
}

\begin{abstract}
${ }^{1}$ Orgnanic Materials and Fiber Engineering Department, Chonbuk National University, Jeonju, Republic of Korea; ${ }^{2} \mathrm{Chemical} \mathrm{Engineer-}$ ing Department, Faculty of Engineering, El-Minia University, El-Minia, Egypt; ${ }^{3}$ Technical University of Denmark, DTU Food, Lyngby, Denmark; ${ }^{4}$ Department of Chemistry, College of Science, King Saud University, Riyadh, Saudi Arabia.

Email: ${ }^{*}$ nasser@jbnu.ac.kr
\end{abstract}

Received May 10 ${ }^{\text {th }}, 2011$; revised May 20 $0^{\text {th }}, 2011$; accepted May $27^{\text {th }}, 2011$.

\begin{abstract}
Doping of titanium dioxide nanofibers by silver nanoparticles revealed distinct improvement in the photocatalytic activity; however other influences have not been investigated. In this work, effect of sliver-doping on the crystal structure, the nanofibrous morphology as well as the photocatalytic activity of titanium oxide nanofibers has been studied. Silver-doped $\mathrm{TiO}_{2}$ nanofibers having different silver contents were prepared by calcination of electrospun nanofiber mats consisting of silver nitrate, titanium isopropoxide and poly(vinyl acetate) at $600{ }^{\circ} \mathrm{C}$. The results affirmed formation of silver-doped $\mathrm{TiO}_{2}$ nanofibers composed of anatase and rutile when the silver nitrate content in the original electrospun solution was more than $3 \mathrm{wt} \%$. The rutile phase content was directly proportional with the $\mathrm{AgNO}_{3}$ concentration in the electrospun solution. Negative impact of the silver-doping on the nanofibrous morphology was observed as increase the silver content caused to decrease the aspect ratio, i.e. producing nanorods rather nanofibers. However, silver-doping leads to modify the surface roughness. Study of the photocatalytic degradation of methylene blue dye clarified that increase the silver content strongly enhances the dye oxidation process.
\end{abstract}

Keywords: Titanium Oxide Nanofibers, Nanostructured Ceramics, Inorganic Materials, Crystal Structure, Electrospinning

\section{Introduction}

Enormous efforts have been devoted to the research of $\mathrm{TiO}_{2}$ material, which has led to many promising applications in areas ranging from photovoltaics and photocatalysis to photo-/electrochromics and sensors [1-5]. New physical and chemical properties emerge when the size of the $\mathrm{TiO}_{2}$ becomes smaller and smaller, and down to the nanometer scale. The main advantage of the nanostructural materials is the high surface to volume ratio, however much literature indicated that the one-dimensional (ID) nanostructures have special characteristics due to the axial ratio aspect [6-8]. Among the ID shapes, nanofibers have novel features because of the long axial ratio; consequently good reports were introduced about fabrication of $\mathrm{TiO}_{2}$ nanofibers/nanotubes [9-11]. Recently, metal-doping strategy has been invoked to im- prove the physical and chemical properties of $\mathrm{TiO}_{2}$ nanoparticles/nanofibers [12-14]. Noble metal incorporation into the titania dielectric provides an absorption feature due to the surface Plasmon resonance (SPR) occurring over the visible range of the spectrum [15]. In particular, silver and gold metals are the most popular materials due to the strong SPR character [16]. Metal-doping may also have an influence on the titania crystal structure leading to distinct improving in the application fields $[13,17]$. Here, we study effect of the silver-doping on the crystal structure, the morphology and the photocatalytic activity of $\mathrm{TiO}_{2}$ nanofibers. Several techniques have been introduced to fabricate the nanofibers, however electrospinning is the most widely utilized process due to its simplicity, high yield and low cost [18-21]. Accordingly, silver-doped $\mathrm{TiO}_{2}$ nanofibers were prepared by calcination of electrospun mats consisting of silver nitrate, tita- 
nium isopropoxide and poly(vinyl acetate) at $600^{\circ} \mathrm{C}$. Control of silver content in the prepared nanofibers could be achieved by modifying the silver nitrate concentration in the original electrospun solution. Physiochemical characterizations indicated that the silver content does have distinct influences on the morphology, crystal structure and photocatalytic activity of the $\mathrm{TiO}_{2}$ nanofibers.

\section{Experimental Section}

\subsection{Materials}

Silver nitrate (99.8 assay), methylene blue dihydrate (95.0 assay), acetic acid (99.7 assay) and N,N-dimethylformamide (DMF, 99.5 assay) were obtained from Showa, Co. Japan. Titanium (1V) isopropoxide (Ti(Iso)), 98.0 assay) was purchased from Junsei Co. Ltd., Japan. Poly(vinyl acetate) (PVAc, MW $=500,000 \mathrm{~g} / \mathrm{mol}$ ) obtained from Aldrich USA. These materials were used without any further purification.

\subsection{Electrospinning Set-up}

A sol-gel composed of titanium isopropoxide (Ti(Iso)) and poly(vinyl acetate) (14\% wt, in N,N-dimethylformamide; DMF) was prepared by mixing these ingredients for $10 \mathrm{~min}$, and then few drops of acetic acid were added till the solution became transparent. The final solution contained $60 \mathrm{wt} \% \mathrm{Ti}$ (Iso). Ten gram of the prepared solution was mixed with silver nitrate/DMF solution $\left(\mathrm{AgNO}_{3}\right.$ was dissolved in a minimum amount of DMF) to prepare the silver-doped titanium oxide nanofibers [14], several solutions containing different contents of silver nitrate have been prepared. In the electrospinning process, a high voltage power supply (CPS-60 K02V1, Chungpa EMT Co., Republic of Korea) was used as a source of electric field. The sol-gel was supplied through a plastic syringe attached to a capillary tip. A graphite rod connected with the positive electrode (anode) was inserted into the sol-gel while the negative one (cathode) was attached to a metallic collector covered with polyethylene sheet. Briefly, the solutions were electrospun at $6 \mathrm{kV}$ voltage and $15 \mathrm{~cm}$ working distance (the distance between the needle tip and the collector). The formed nanofiber mats were initially dried for $24 \mathrm{~h}$ at $80^{\circ} \mathrm{C}$ under vacuum and then calcined in air atmosphere at $600^{\circ} \mathrm{C}$ for $1 \mathrm{~h}$ with a heating rate of $5^{\circ} \mathrm{C} / \mathrm{min}$.

The photocatalytic degradation of methylene blue dye was carried out in a simple photo reactor. The reactor was made of glass (1000 $\mathrm{ml}$ capacity, $23 \mathrm{~cm}$ height and $15 \mathrm{~cm}$ diameter), covered with alumina foil and equipped with $365 \mathrm{~nm}$ (wavelength radiation) ultra-violet lamp. The initial dye solution and the photocatalyst were placed in the reactor and continuously stirred for complete mixing during the photocatalytic reaction. Typically, 100 $\mathrm{ml}$ of the dye solution (10 ppm, concentration) and 50 $\mathrm{mg}$ of the catalyst were used. At specific time intervals, 2 $\mathrm{ml}$ samples were withdrawn from reactor and centrifuged to separate the nanofibers (catalyst), and then the absorbance intensity was measured at wavelength of 664 $\mathrm{nm}[14]$.

\subsection{Characterization}

The phase and crystallinity were characterized by using Rigaku X-ray diffractometer (Rigaku Co, Japan) with $\mathrm{Cu}$ $\mathrm{K} \alpha(\lambda=1.54056 \AA)$ radiation over a range of $2 \theta$ angles from 20 to 800 . Surface morphology of the nanofibers was studied by JEOL JSM-5900 scanning electron microscope, JEOL Ltd, Japan and field-emission scanning electron microscope (FESEM, Hitachi S-7400, Japan). High resolution image was observed by JEOL JEM 2010 transmission electron microscope (TEM) operating at $200 \mathrm{kV}$, JEOL Ltd., Japan. The concentration of the dyes during the photodegradation study was investigated by spectroscopic analysis using HP 8453 UV-visible spectroscopy system, Germany. The spectra obtained were analyzed by HP ChemiStation software 5890 series.

\section{Results and Discussion}

Titanium oxide has three popular crystal structures; antase, rutile and brookite. Anatase is a polymorph with the two other minerals. The minerals rutile and brookite as well as anatase all have the same chemical formula, $\mathrm{TiO}_{2}$, but they have different structures. Rutile is the more common and the more well known mineral of the three, while anatase is the rarest. Anatase shares many of the same or nearly the same properties as rutile such as luster, hardness and density. However due to structural differences, anatase and rutile differ slightly in the crystal habit. The phase change from anatase polymorph of titania to rutile one has been the subject of considerable interest and the focus of many groups' activities over the years. Figure 1 shows the effect of silver-doping on the crystal structure of the obtained powder after the calcination process. In case of silver-free solution, the results affirm formation of pure anatase titanium dioxide, existence of strong diffraction peaks at $2 \theta$ values of $25.09^{\circ}$, $37.65^{\circ}, 38.44^{\circ}, 47.89^{\circ}, 53.89^{\circ}, 55.07^{\circ}, 62.40^{\circ}, 68.70^{\circ}$, $70.04^{\circ}$ and $75.00^{\circ}$ corresponding to the crystal planes of (101), (004), (112), (200), (105), (211), (204), (220), (220) and (215), respectively indicates formation of anatase titanium dioxide [JCPDS card no 21-1272]. Addition of small amount of silver nitrate $(2 \mathrm{wt} \%)$ did not affect the crystal structure of the titania, however pure silver metal was formed due to decomposition of the silver nitrate upon heating. The extra peaks at $2 \theta$ values of $38.11^{\circ}$, $44.29^{\circ}, 64.43^{\circ}$ and $77.48^{\circ}$, corresponding to the crystal planes of (111), (200), (220) and (311), respectively affirm 


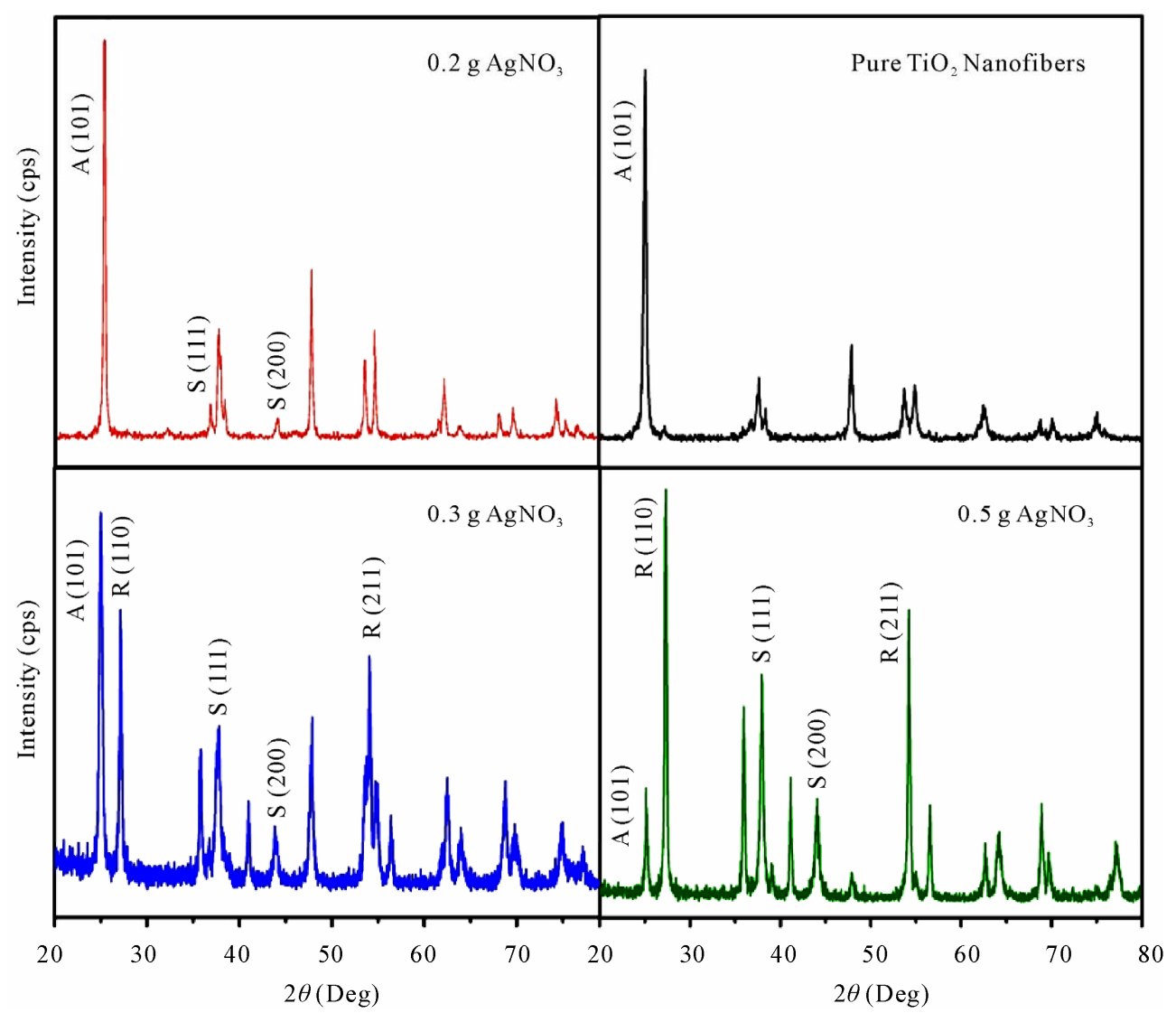

Figure 1. Effect of silver content on the crystal structure of $\mathrm{TiO}_{2}$.

presence of silver metal [JCPDS card no 04- 0783].

In Figure 1, the main peaks of anatase and silver were marked as $\mathrm{A}$ and $\mathrm{S}$, respectively. Increase the silver nitrate content to $3 \mathrm{wt} \%$ in the original electrospun solution led to partial phase change in the titania crystal structure. The standard peaks of rutile can be observed at $2 \theta$ values of $27.45^{\circ}, 36.09^{\circ}, 41.23^{\circ}, 54.32^{\circ}, 56.64^{\circ}$, and $69.08^{\circ}$ corresponding to the crystal planes of (110), (101), (111), (211), (220), and (301), respectively [JCPDS card no 21-1272]. Interestingly, the results indicate that increase the amount of silver enhances formation rutile phase as shown in Figure 1 (the main rutile peaks were marked by $\mathrm{R}$ letter). Addition of $5 \mathrm{wt} \%$ silver nitrate led to increase the rutile content to be higher than the anatase. According to the XRD data, the ratio of rutile to anatase is $\sim 75 \%$ and $440 \%$ when the added silver nitrate was 3 $\mathrm{wt} \%$ and $5 \mathrm{wt} \%$, respectively. The electrospinning technique involves the use of a high voltage to charge the surface of a polymer solution droplet and thus to induce the ejection of a liquid jet through a spinneret. Due to bending instability, the jet is subsequently stretched by many times to form continuous, ultrathin fibers. It is widely used for production of many polymeric nanofibers. Moreover, the electrospinning process has been exploited to produce metal oxides nanofibers by calcination of electrospun mats obtained from completely miscible sol-gel solutions. Electrospinning of a sol-gel composed of $\mathrm{Ti}(\mathrm{IsO})$ and PVAc/DMF solution has been carried out in our lab in previous study [22], the resultant electrospun nanofibers have good morphology. Addition of silver nitrate to the sol-gel does not affect the morphology [14]. Accordingly, well morphology electrospun nanofibers mats were obtained from $\mathrm{AgNO}_{3} / \mathrm{Ti}$ (Iso)/PVAc solutions at all the utilized $\mathrm{AgNO}_{3}$ contents (data are not shown). Figure 2 shows the morphology of the resultant powder after the calcination process. As can be observed in Figures 2(a) and 2(b) which demonstrate the obtained product from silver-free electrospun solution, well and smooth $\mathrm{TiO}_{2}$ nanofibers were obtained, these nanofibers composed of pure anatase as could be concluded from the XRD results (Figure 1). Incorporation of small amount of silver does not have considerable impact on the nanofibrous morphology as shown in Figure 2(c) and 2(d) which represent the FE-SEM images of the powder obtained from calcination of an electrospun solution containing $2 \mathrm{wt}^{\mathrm{O}} \mathrm{\textrm {AgNO } _ { 3 }}$. However, the surface became little rough (Figure 2(d)). Figure 3 demonstrates the TEM analysis results for $\mathrm{TiO}_{2}$ nanofibers originated from 


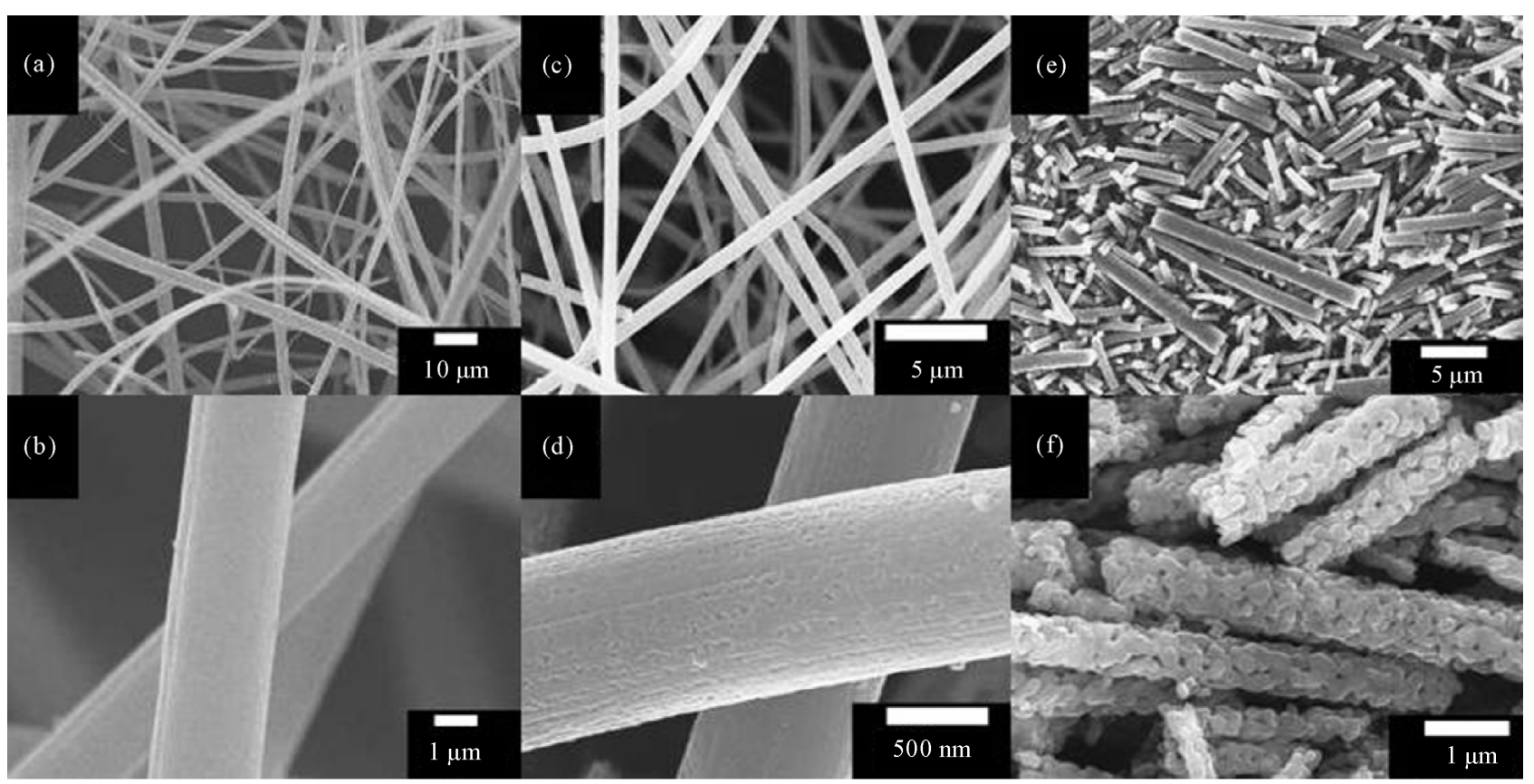

Figure 2. Effect of silver content on the nanofibrous morphology of $\mathrm{TiO}_{2}$.

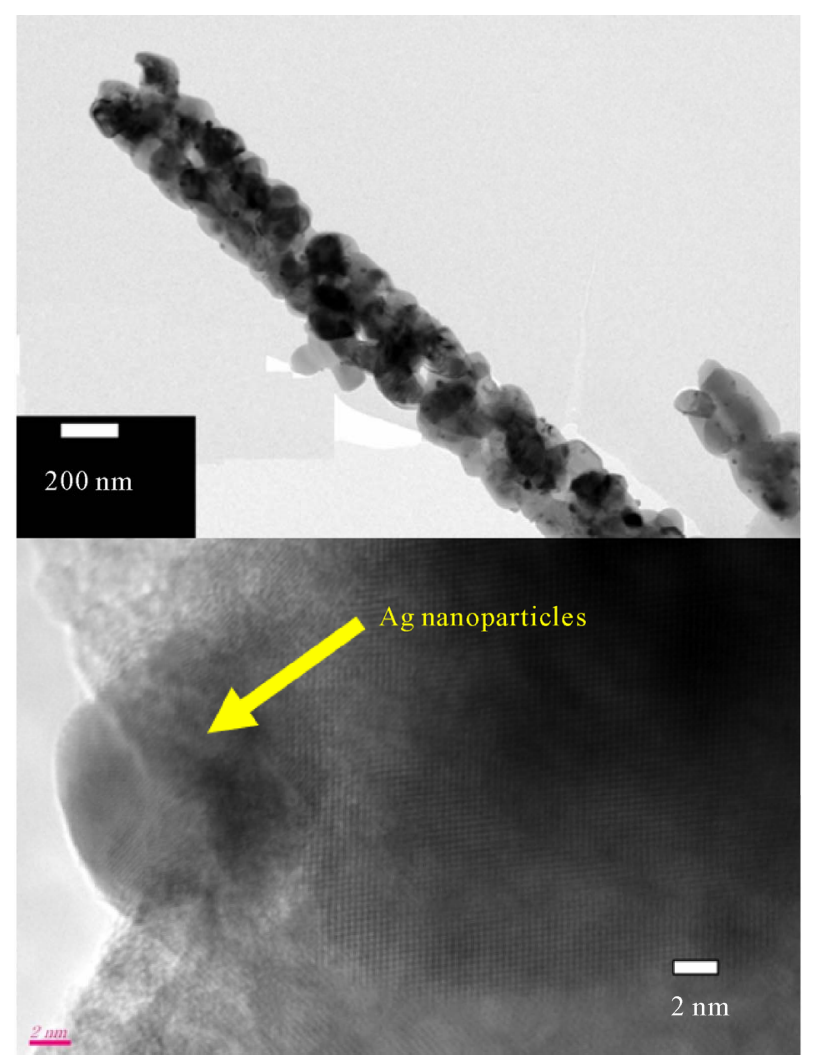

Figure 3. TEM image for the $\mathrm{TiO}_{2}$ nanofibers originated from electrospun solution containing 2 wt \% silver nitrate.

an electrospun solution containing $2 \mathrm{wt} \%$ silver nitrate. Due to the large atomic radius of the silver atom, they cannot interpenetrate in $\mathrm{TiO}_{2}$ crystals to form solid solution alloy. Therefore, silver metal distributes in the form of separated nanoparticles as shown in the normal TEM and HR TEM images. Of course, the average size of those nanoparticles is directly proportional with the amount of silver. Therefore, more sharp and strong peaks were observed in the XRD spectra representing the nanofibers containing high silver content. This might be an acceptable explanation of obtaining nanorods rather than nanofibers when the amount of the utilized silver nitrate was $3 \mathrm{wt} \%$ (Figure 2(e) and 2(f)), in other words, the big size silver nanoparticles led to break the nanofibers. However, the surface became much rougher (Figure 2(f)) compared with the pristine and low silver content $\mathrm{TiO}_{2}$ nanofibers. More increase in the silver nitrate content in the electrospun solution led to complete annihilation of the 1D structure. Much literature has been reported explaining the positive effect for silver incorporation on the photocatalytic of $\mathrm{TiO}_{2}$ nanostructures. Generally, these studies have drawn this conclusion. The Ag nanoparticles deposited on $\mathrm{TiO}_{2}$ surface act as electron acceptors, enhancing the charge separation of electrons and holes and consequently the transfer of the trapped electron to the adsorbed $\mathrm{O}_{2}$. The sufficient dye molecules are adsorbed on the surface of $\mathrm{Ag}-\mathrm{TiO}_{2}$ more than on the $\mathrm{TiO}_{2}$ surface, increasing the photoexcited electron transfer from the sensitized dye molecule to the conduction band of $\mathrm{TiO}_{2}$ and consequently increasing the electron transfer to the adsorbed $\mathrm{O}_{2}$. Figure 4 affirms this conclusion. As shown in Figure $\mathbf{4}$ which represents the effect of silver content 


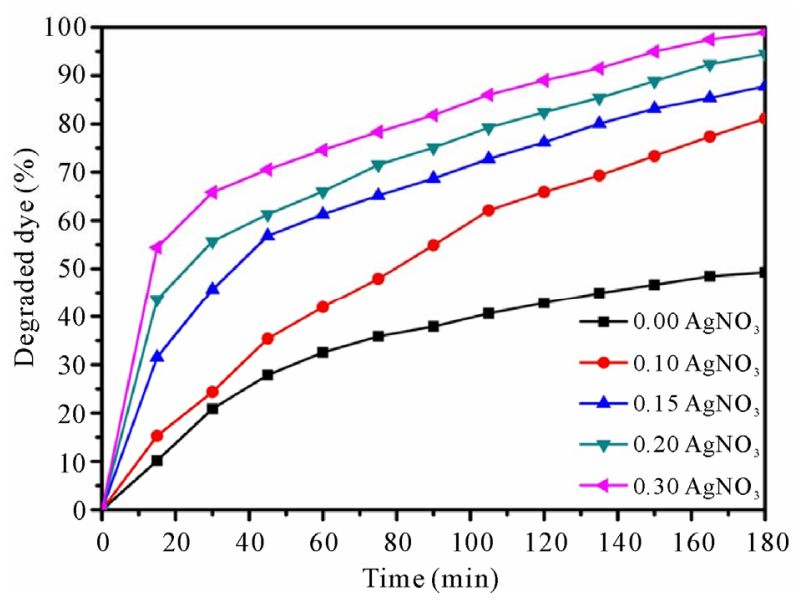

Figure 4. Effect of silver content on the photocatalytic activity of $\mathrm{TiO}_{2}$ nanofibers.

on photcatalytic degradation of methylene blue dye, the rate of degradation enhances with increasing the silver content in the $\mathrm{TiO}_{2}$ nanofibers. Moreover, the surface roughness might have an influence due to increase the total surface area.

\section{Conclusions}

Calcination of electrospun nanofiber mats composed of silver nitrate, titanium isopropxide and poly(vinyl acetate) in air at $600^{\circ} \mathrm{C}$ leads to produce silver-doped $\mathrm{TiO}_{2}$ nanofibers having both of antase and rutile phases if the silver nitrate content is more than $3 \mathrm{wt} \%$. Rutile phase content can be increased by increasing the silver content in the original electrospun solution. However, the nanofibrous morphology is strongly affected by the silver-content, excess silver causes to destroy the 1D structure. Silver-doping of the titanium oxide nanofibers greatly enhances the photocatalytic activity of this interesting material.

\section{Acknowledgements}

This paper was supported by research funds of chonbuk National University 2010. We thank Mr. T. S. Bae and J. C. Lim, KBSI, Jeonju branch, and Mr. Jong-Gyun Kang, Centre for University Research Facility, for taking highquality FESEM and TEM images, respectively.

\section{REFERENCES}

[1] X. Chen and S. D. Mao, "Titanium Dioxide Nanomaterials: Synthesis, Properties, Modifications, and Applications," Chemical Review, Vol. 107, No. 7, 2007, pp. 28912959. doi:10.1021/cr0500535

[2] M. Grätzel, "Photoelectrochemical Cells," Nature, Vol. 414, 2001, pp. 338-344.

[3] A. Millis and S. Le Hunte, "An Review of Semiconductor
Photocatalysis," Journal of Photochemistry and Photobiology, Vol. 108, No. 1, 1997, pp. 1-35.

[4] S. Xu, J. Ng, X. Zhang, H. Bai and D. D. Sun, “Adsorption and Photocatalytic Degradation of Acid Orange 7 over Hydrothermally Synthesized Mesoporous $\mathrm{TiO}_{2}$ Nanotube," Colloids and Surfaces A, Vol. 379, No. 1, 2011, pp. 169-175.

[5] C. K. Lee, C. C. Wang, L. C. Juang, M. D. Lyu, S. H. Hung and S. S. Liu, "Effects of Sodium Content on the Microstructures and Basic Dye Cation Exchange of Titanate Nanotubes," Colloids and Surfaces A, Vol. 317, No. 1, 2008, pp. 164-173.

[6] M. Fraune, U. Rüdiger and G. Güntherodt, "Size Dependence of the Exchange Bias Field in Ni/NiO Nanostructures," Applied Physics Letters, Vol. 77, 2000, pp. 3815-3817. doi:10.1063/1.1330752

[7] N. A. M. Barakat, B. Kim, S. J. Park, Y. Jo, M. H. Jung and H. Y. Kim, "Cobalt Nanofibers Encapsulated in Graphite Shell with Thermally Independent Magnetic Properties," Journal of Materials Chemistry, Vol. 19, No. 39, 2009, pp. 7371-7378. doi:10.1039/b904669k

[8] N. A. M. Barakat, K. A. Khalil, I. H. Mahmoud, M. A. Kanjwal, F. A. Sheikh and H. Y. Kim, "CoNi Bimetallic Nanofibers by Electrospinning: Nickel-Based Soft Magnetic Material with Improved Magnetic Properties," Journal of Physical Chemistry C, Vol. 114, No. 37, 2010, pp. 15589-15593.

[9] D. Li and Y. Xia, "Fabrication of Tatania Nanofibers by Electrospinning," Nano Letters, Vol. 3, No. 4, 2003, pp. 555-560. doi: $10.1021 / \mathrm{n} 10340390$

[10] Y. Z .Yuan and B. L. Su, "Titanium Oxide Nanotubes, Nanofibers and Nanowires," Colloids and Surfaces A, Vol. 241, No. 1-3, 2004, pp. 173-183.

[11] A. DiPaola, G. Marci, L. Palmisano, M. Schiavello, K. Uosaki, S. Ikeda and B. Ohtani, "Preparation of Polycrystalline $\mathrm{TiO}_{2}$ Photocatalysts Impregnated with Various Transition Metal Ions: Characterization and Photocatalytic Activity for the Degradation of 4-Nitrophenol," Journal of Physical Chemistry B, Vol. 106, 2002, pp. 637-645. doi:10.1021/jp0130741

[12] N. A. M. Barakat, M. S. Khill and H. Y. Kim, "Preparation of MnO Nanofibers by Novel Hydrothermal Treatment of Manganese Acetate/PVA Electrospun Nanofiber Mats," Materials Science and Engineering B, Vo. 162, 2009, pp. 205-208.

[13] J. Arbiol, J. Cerdá, G. Dezanneau, A. Cirera, F. Peiró, A. Cornet and J. R. Morante, "Effects of Nb Doping on the $\mathrm{TiO}_{2}$ Anatase-to-Rutile Phase Transition," Journal of Applied Physics, Vol. 92, No. 2, 2002, pp. 853-861. doi:10.1063/1.1487915

[14] M. A. Kanjwal, N. A. M. Barakat, F. A. Sheikh, M. S. Khil and H. Y. Kim, "Functionalization of Electrospun Titanium Oxide Nanofibers with Silver Nanoparticles: Strongly Effective Photocatalyst," International Journal of Applied Ceramics Technology, Vol. 7, Suppl. 1, 2010, pp. E54-E63.

[15] N. A. M. Barakat, K. D. Woo, M. A. Kanjwal, K. E. Choi, 
M. S. Khil and H.Y. Kim, "Surface Plasmon Resonances, Optical Properties and Electrical Conductivity Thermal Hystersis of Silver Nanofibers Produced by Electrospinning Technique," Langmuir, Vol. 24, 2008, pp. 1198211987.

[16] T. Nikolajsen, T. Leosson, K. Salakutdinov and S. Bozhevolnyi, "Surface Plasmon Polariton Based Modulators and Switches Operating at Telecom Wavelengths," Applied Physics Letters, Vol. 85, 2004, pp. 5833-5836. doi:10.1063/1.1835997

[17] K. H. Ko, Y. C. Lee and Y. J. Jung, "Enhanced Efficiency of Dye-Sensitized $\mathrm{TiO}_{2}$ Solar Cells (DSSC) by Doping of Metal Ions," Journal of Colloid and Interface Science, Vol. 283, No. 2, 2005, pp. 482-487. doi:10.1016/j.jcis.2004.09.009

[18] W. Sigmund, J. Yuh, H. Park, V. Maneeratana, G. Pyrgiotakis, A. Daga, J. Taylo and J. C. Nino, "Processing and Structure Relationships in Electrospinning of Ceramic Fiber Systems," Journal of American Ceramic So- ciety, Vol. 89, No. 2, 2006, pp. 395-407. doi:10.1111/j.1551-2916.2005.00807.x

[19] N. A. M. Barakat and H. Y. Kim, "Metal Oxides Nanostructures and Their Applications," American Scientific Publisher, Stevenson Ranch, 2009.

[20] M. S. Islam and M. R. Karim, "Fabrication and Characterization of Poly(Vinyl Alcohol)/Alginate Blend Nanofibers by Electrospinning Method," Colloids and Surfaces A, Vol. 366, No. 1-3, 2010, pp. 135-140.

[21] A. R. Abbasi and A. Morsali, "Ultrasound-Assisted Coating of Silk Yarn with Silver Chloride Nanoparticles," Colloids and Surfaces A, Vol. 371, No. 1, 2010, pp. 113118.

[22] B. Ding, C. K. Kim, H. Y. Kim, M. S. Khil and S. Park, "Titanium Dioxide Nanofibers Prepared by Using Electrospinning Method," Fibers and Polymers, Vol. 5, 2005, pp. 105-109. 